\title{
TEMA -2016: Evolocumab y sus posibles beneficios para una población en riesgo
}

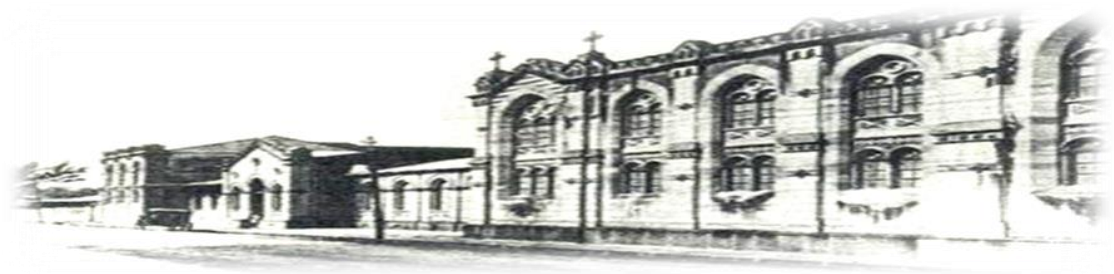

ISSN

Hospital San Juan de Dios, San José, Costa Rica. Fundado en 1845

Recibido: $\quad 05 / 09 / 2016$

Aceptado: $\quad 06 / 10 / 2016$

${ }^{1}$ Dra. Jimena Araya Vargas

${ }^{2}$ Dr. Mario Bolaños Arrieta

${ }^{1}$ Licenciada en Medicina y Cirugía. Universidad de Costa Rica. Correo electrónico:

${ }^{2}$ Médico especialista en Cardiología, Jefe de Clínica de Cardiología del Hospital México. Correo electrónico:

\section{RESUMEN}

La disminución en los niveles de LDL sérico con estatinas ha demostrado reducir las complicaciones y mortalidad por enfermedad cardiovascular. Sin embargo, $10-20 \%$ de los pacientes muestran intolerancia a éstas o resistencia para lograr la meta de LDL como en pacientes con hipercolesterolemia familiar. Actualmente se han desarrollado estudios en búsqueda de medicamentos alternativos para pacientes con hipercolesterolemia severa, como es el caso del Evolocumab. Éste es un anticuerpo monoclonal dirigido contra PCSK9, que permite la reducción en la concentración sérica de LDL. Los estudios demostraron una disminución estadísticamente significativa de hasta un $66 \%$ en los grupos tratados con Evolocumab, asociado o no a estatinas, en comparación con placebo y/o ezetimibe. Mostrando ser efectiva en un lapso de 12 semanas. Aunque fue bien tolerado se relacionó con efectos adversos, sin embargo su incidencia fue similar es comparación al grupo control. El problema de estos estudios se debe a in- consistencias en el diseño de éstos, así como conflicto de interés por parte sus autores. Aunque se ha demostrado la efectividad del Evolocumab en la disminución del LDL, es necesario realizar una mayor investigación que permita comprender su eficacia y seguridad a largo plazo, así como demostrar una posible reducción en la mortalidad. Hasta el momento, el Evolocumab es una alternativa terapéutica en aquellas poblaciones con hipercoleterolemia familiar que no logran alcanzar niveles óptimos de LDL

\section{PALABRAS CLAVE}

Evolocumab, PCSK9, hipercolesterolemia familiar, medicamentos alternativos.

\section{ABSTRACT}

The decrease in serum LDL with statins has been shown to reduce complications and mortality from cardiovascular disease. However, 10-20\% of these patients show intolerance or resistance to 
achieve the goal of LDL like in patients with familial hypercholesterolemia. Studies have now been developed in search for alternative drugs for patients with severe hypercholesterolemia such as the Evolocumab. This is a monoclonal antibody directed against PCSK9, which allows reduction in serum LDL. Studies show a statistically significant decrease of up to $66 \%$ in the groups treated with Evolocumab, with or without a statin, versus placebo and / or ezetimibe. Proving to be effective over a period of 12 weeks. Although it was well tolerated, associated with side effects. However the incidence was similar is compared to the control group. The problem with these studies is due to inconsistencies in designing these and conflict of interest by their authors. Although it has been shown Evolocumab effectiveness in reducing LDL, it's necessary to perform more research to understand its effectiveness and long-term safety and demonstrate a possible reduction in mortality. So far, the Evolocumab is a therapeutic alternative in those populations with family hipercoleterolemia who fail to achieve optimal levels of LDL.

\section{KEY WORDS}

Evolocumab, PCSK9, familial hypercholesterolemia, alternative drugs.

\section{INTRODUCCIÓN}

La principal causa de muerte en América, así como en Australia y el norte de Europa, se debe a enfermedad cardiovascular. De allí radica la importancia de campañas en prevención primaria, así como un adecuado y pronto control de las patologías crónicas del paciente, con el fin de disminuir su mortalidad por algún evento cardiovascular. ${ }^{1}$

Se sabe que mantener niveles óptimos de LDL según la estratificación de riesgo de cada paciente, disminuye el riesgo cardiovascular. Si bien es cierto, las guías internacionales recomiendan el uso de estatinas como tratamiento de primera línea en las hiperlipidemias; algunos pacientes no toleran este medicamento a altas dosis (16-53\%) como es el caso de aquellos pacientes con hipercolesterolemia familiar, o no toleran sus efectos secundarios $(10-20 \%)^{2}$
El fenotipo en aquellos pacientes con hipercolesterolemia severa (LDL >190 mg/dl), se debe en su mayoría a mutaciones autosómicas dominantes a nivel de los genes que codifican para el receptor de LDL, la apolipoproteína B, o de la PSCK9. Los otros casos tienen un origen poligénico en genes no identificados, o se deben a causas epigenéticas.

La hipercolesterolemia familiar (HF) se debe a anormalidades a nivel del receptor de LDL. Esta patología a su vez puede clasificarse en homocigota si se debe a mutaciones que afectan ambos alelos de los genes que codifican para el receptor de LDL, heterocigota compuesta si comprende una mutación diferente en cada alelo, o heterocigota si solo posee un alelo mutado. ${ }^{3}$

Aunque dependiendo del genotipo de cada paciente así será el grado de alteración la vía, y los niveles de LDL serán mayores o menores según corresponda; la realidad es que el riesgo cardiovascular está determinado por la exposición a la hipercolesterolemia (fenotipo) independientemente de su origen genético. ${ }^{4}$

\section{DISCUSIÓN}

Evolocumab, el tratamiento del futuro

Debido a que en poblaciones con hipercolesterolemia severa, particularmente aquellos con hipercolesterolemia familiar, la monoterapia con estatinas generalmente no es suficiente, o en pacientes intolerantes a éstas, se necesitan múltiples fármacos, hasta tres, e inclusive la aféresis para lograr acercarse a la meta, especialmente en pacientes de alto riesgo cardiovascular; en los últimos años se han desarrollado estudios enfocados en fármacos que puedan beneficiar a este sector de la población, como es el caso del Evolocumab. ${ }^{5}$ El Evolocumab (AMG-145) es un anticuerpo monoclonal (Ig G2) dirigido contra PCSK9 (Proprotein Convertase Subtilisin/Kexin type 9) con el que se desarrollaron estudios clínicos. ${ }^{6}$

La PCSK9 es una convertasa sintetizada a nivel de hígado e intestino delgado, la cual se encarga de disminuir el reciclaje del receptor de LDL a nivel hepático, al unirse a éste y cambiar su conformación, aumentando su degradación a nivel lisosomal, e incrementando la concentración sérica de LDL. ${ }^{7}$ Las mutaciones con ganancia de función a 
nivel de esta proteína favorece el fenotipo de hipercolesterolemia severa. Si por el contrario se presenta una mutación con pérdida de la función, actúa como protector cardiovascular. Es por ello que la inhibición de la PCSK9 por medio del Evolocumab, incrementa la expresión de receptores de LDL hepático, y por ende, disminuye los niveles de LDL circulante. ${ }^{8}$

\section{Estudios Relevantes}

Se han realizado múltiples ensayos clínicos en un lapso de 12 semanas. Los pacientes se dividieron en grupos expuestos a Evolocumab como monoterapia, así como asociados o no a estatinas y/o ezetimibe, además del grupo placebo. Se aplicaron diversas dosis de medicamentos en los diferentes estudios $(70 \mathrm{mg}, 105 \mathrm{mg}$ ó $140 \mathrm{mg}$ cada dos semanas; ó 280, 350 o 420 mg mensualmente). ${ }^{9}$

Entre los estudios cabe recalcar el LAPLACETIMI 57, en donde de los 631 pacientes del estudio, un $45 \%$ (284) tenía de alto riesgo cardiovascular. De ellos, a los que se les administró el medicamento cada 2 semanas, un $90 \%$ llegó a los niveles meta de LDL (<70 mg/dl); mientras que en el grupo que lo recibía cada 4 semanas, un $70 \%$ llegó a este objetivo. Tomando en cuenta que solo hasta el 35\% de los pacientes de alto riesgo llegan a alcanzar el propósito según el ATP III, el tratamiento sinérgico entre las estatinas y el AMG 145 es una buena opción para esta población. Sin embargo, aún no existen estudios que permitan evidenciar si realmente el Evolocumab disminuye la mortalidad. ${ }^{10,11}$

Otros estudios importantes por razones distintas fueron el MENDEL y GAUSS, ambos en fase II y III, los cuales fueron los únicos en valorar en efecto del Evolocumab como monoterapia o asociado a ezetimibe. A favor del MENDEL, tiene que es de los estudios con mayor tamaño de muestra expuesta al medicamento comparado con los demás. Sin embargo, una limitante del mismo es que los pacientes escogidos tenían un bajo riesgo cardiovascular; y en el MENDEL-2 un Framingham $<10 \%$. Teniendo en consideración que la mayoría de las personas que se vería beneficiada de este tratamiento tienen un riesgo cardiovascular alto, este estudio no es útil para valorar su efecto en esta futura población meta. ${ }^{2,12}$
Por otro lado, en el estudio GAUSS se incluyeron 157 pacientes intolerantes a las estatinas, con LDL $>100 \mathrm{mg} / \mathrm{dl}$. Después de 12 semanas de tratamiento, se redujo el LDL entre un $41 \%$ y un $51 \%$ en aquellos que solo recibieron Evolocumab. Los que además recibieron ezetimibe redujeron el LDL en $63 \%$. Y los que únicamente recibían este último, un $14 \%$. Ventajas de este estudio con respecto al MENDEL: $50 \%$ de la muestra tenían un riesgo de moderado a alto, $17 \%$ enfermedad arterial coronaria, $7 \%$ enfermedad cerebro vascular o enfermedad arterial periférica. Desventaja: el tamaño de la muestra respecto del anterior es inferior, lo cual también es un problema constante en la mayoría de investigaciones. ${ }^{13,14}$

Otro estudio importante fue el Rutherford en fase 2 y 3 , cuya población tenía el diagnóstico clínico de hipercolesterolemia familiar, junto con un LDL igual o mayor a $100 \mathrm{mg} / \mathrm{dl}$, y que previamente recibían estatinas con o sin ezetimibe. Al final de 12 semanas, se obtuvo una reducción del LDL de hasta un $61 \% .^{15,16}$ Además demostró que en aquellos pacientes con hipercolesterolemia heterocigota, el screening genético no es relevante para medir efectividad del tratamiento; como sí lo es en el caso de homocigotas, en los cuales se obtienen mayores reducciones de LDL respecto a la basal en contraste con heterocigotas, lo cual también fue demostrado en el TESLA. ${ }^{9}$ En este último se documentó que la reducción del LDL fue mayor en aquellos portadores de una mutación de defecto en uno o dos alelos del receptor, versus el grupo placebo. En los pacientes con una sola mutación negativa o de defecto, se obtuvo una disminución menor en los niveles de LDL comparados con la población anterior, aunque igualmente significativa en relación al placebo. ${ }^{17}$

En 2015 se publicaron los resultados del programa OSLER (Open-Label Study of Long-Term Evaluation against LDL Cholesterol), cuyo objetivo fue valorar seguridad y eficacia a largo plazo. Este estudio reclutó un total de 4465 pacientes que habían formado parte de las investigaciones anteriores, y se dividieron en OSLER-1 (para aquellos pacientes que completaron los estudios fase 2) y OSLER-2 (para aquellos que completaron los estudios fase 3). En total, 2976 recibieron Evolocumab más estatinas y 1489 únicamente estatinas. A los pertenecientes a OSLER-1 se les administraron $420 \mathrm{mg}$ de Evolocumab subcutánea cada mes; 
mientras que los de OSLER-2 recibieron una dosificación $140 \mathrm{mg}$ cada 2 semanas y $420 \mathrm{mg}$ cada mes; y se les dio seguimiento durante 11.1 meses. Y la reducción en los niveles de LDL fue comparable a los estudios anteriores. ${ }^{18}$

Respecto a la incidencia de efectos adversos, fue similar entre los grupos, $69.2 \%$ en el grupo tratado con evolocumab y $64.8 \%$ en los que recibieron terapia convencional. ${ }^{18}$ Los más frecuentes fueron la nasofarigitis, náuseas y cefalea, mialgias así como reacción en el sitio de punción. También se evidenció que la elevación de AST/ALT tres veces más sobre el límite superior, fue semejante entre los grupos que recibieron Evolocumab y los grupos control. Igualmente no hubo diferencia estadísticamente significativa entre ambos grupos respecto al aumento de la $\mathrm{CK} .{ }^{15} \mathrm{Un}$ dato interesante es que por primera vez se documentan eventos adversos neurocognitivos, aunque fue menor al $1 \%$. Entre los eventos adversos serios se presentaron en porcentajes iguales entre el grupo control versus los que recibieron Evolocumab $(7.5 \%)$. No se detectaron anticuerpos neutralizantes durante el periodo de investigación. ${ }^{18}$

En general los estudios demostraron una disminución estadísticamente significativa de hasta un $66 \%$ en las concentraciones plasmáticas de LDL en los grupos tratados con evolocumab, asociado o no a estatinas, en comparación con placebo y/o ezetimibe. ${ }^{11}$ La reducción fue dosis dependiente y el cambio final respecto a la línea de base fue similar entre los pacientes que recibieron el anticuerpo; sin observarse diferencias significativas basadas en sexo, edad, etnia, niveles séricos previos de LDL o triglicéridos.

La disminución en los niveles de LDL fue mayor entre cada dosificación en comparación con los niveles obtenidos al final de cada intervalo de administración del medicamento. Lo anterior crea la duda de si realmente a largo plazo se mantendría una disminución en la mortalidad (en caso de que llegue a demostrarse tal efecto) aunque se descontinuara el tratamiento, se distanciara el tiempo de administración, o para preservar tal beneficio sería necesario emplearlo de por vida a intervalos constantes. ${ }^{19}$ Lo cual demuestra otra limitante ya que la mayoría de estudios se han realizado únicamente durante 12 semanas.
Otro inconveniente radica en que al ser subcutáneo, el paciente debe inyectarse, lo cual puede provocar resistencia y abandono del mismo. Un punto en contra de estas investigaciones radica en el financiamiento que han recibido por parte de diversas casas farmacéuticas, así como los autores han participado en investigaciones para éstas; ejemplo de ellos son Sanofi, Abbott, Merck Sharp, AstraZeneca y Pfizer, así como Laboratorios Amgen que es la compañía encargada de desarrollar el medicamento Rephata, nombre comercial del AMG-145. ${ }^{16,17}$

\section{CONCLUSIONES}

Cada vez existe mayor evidencia de que el evolocumab podría ser una opción terapéutica eficaz y segura para disminuir los niveles de LDL, en aquellos pacientes con intolerancia a las estatinas o que no logran alcanzar la meta de LDL con las terapias convencionales; específicamente en aquellos con hiperlipidemia familiar homocigota. ${ }^{4}$ Sin embargo, es difícil de evidenciar realmente tales beneficios dado que en muchos de los estudios hay irregularidades en tamaño y escogencia del tipo de población, así como duración del estudio.

Hasta la fecha no existe ninguna investigación que haya determinado su impacto en eventos cardiovasculares y en la mortalidad. Será hasta el 2017 cuando se tiene planteado publicar estos resultados del estudio FOURIER. ${ }^{18}$ El cual además planea extender el tiempo de estudio, y permitiría determinar tanto efectividad como seguridad a largo plazo. De momento, dado el costo aumentado en comparación con las estatinas, el evolocumab es una alternativa en aquellas poblaciones con alto riesgo cardiovascular que no logran alcanzar niveles óptimos de LDL.

\section{BIBLIOGRAFÍA}

1- Mortality and Causes of Death Collaborators. Global, regional, and national age-sex specific all-cause and cause-specific mortality for 240 causes of death, 1990-2013: a systematic analysis for the Global Burden of Disease Study 2013. Lancet 2015; 385: 117-171. 
2- Koren M, Scott R, Kim J, Knusel B, Liu T, Lei $\mathrm{L}$, Bolognese $\mathrm{M}$ et al. Efficacy, safety, and tolerability of a monoclonal antibody to proprotein convertase subtilisin/kexin type 9 as monotherapy in patients with hypercholesterolaemia (MENDEL): a randomised, double-blind, placebo-controlled, phase 2 study. Lancet 2012; 380: 1995-2006.

3- Sniderman A, Tsimikas S, Fazio S. The Severe Hypercholesterolemia Phenotype: Clinical Diagnosis, Management, and Emerging Therapies. J Am Coll Cardiol 2014; 63:1935-47.

4- Thompson G. Managing homozygous familial hypercholesterolaemia from cradle to grave. Atherosclerosis Supplements 2015; 18: 16-20.

5- Catapano A, Papadopoulos N. The safety of therapeutic monoclonal antibodies: Implications for cardiovascular disease and targeting the PCSK 9 pathway. Atherosclerosis 2013; 228:18-28.

6- Dias C, Shaywitz A, Wasserman S, Smith B, Gao B, Stolman D et al. Effects of AMG 145 on Low-Density Lipoprotein Cholesterol Levels Results From 2 Randomized, Double-Blind, Placebo-Controlled, Ascending-Dose Phase 1 Studies in Healthy Volunteers and Hypercholesterolemic Subjects on Statins. J Am Coll Cardiol 2012; 60:1888-98.

7- Farnier M. PCSK9: From discovery to therapeutic applications. Arch Cardiovasc Dis 2014; 107: 58-66.

8- Davidson M. Emerging low-density lipoprotein therapies: Targeting PCSK9 for low-density lipoprotein reduction. J Clin Lipidol 2013; 7: 11-15.

9- Verbeek R, Stoekenbroek R, Hovingh K. PCSK9 inhibitors: Novel therapeutic agents for the treatment of hypercholesterolemia. Eur $\mathrm{J}$ Pharmacol 2015; 763:38-47.

10- Desai N, Giugliano R, Zhou J, Kohli P, Somaratne R, Hoffman E et al. AMG 145, a Monoclonal Antibody Against PCSK9, Facilitates Achievement of National Cholesterol Education Program-Adult Treatment Panel III Low-Density Lipoprotein Cholesterol Goals Among High-Risk Patients An Analysis From the LAPLACE-TIMI 57 Trial (LDL-C Assessment with PCSK9 monoclonaL Antibody Inhibition Combined With Statin
thErapy-Thrombolysis In Myocardial Infarction 57). J Am Coll Cardiol 2014; 63:430-433.

11- Giugliano R, Desai N, Kohli P, Rogers W, Somaratne R, Huang F et al. Efficacy, safety, and tolerability of a monoclonal antibody to proprotein convertase subtilisin/kexin type 9 in combination with a statin in patients with hypercholesterolaemia (LAPLACE-TIMI 57): a randomised, placebo-controlled, dose-ranging, phase 2 study. Lancet 2012; 380: 2007-2017.

12- Koren M, Lundqvist P, Bolognese M, Neutel $\mathrm{J}$, Monsalvo M, Yang J et al. Anti-PCSK9 Monotherapy for Hypercholesterolemia The MENDEL2 Randomized, Controlled Phase III Clinical Trial of Evolocumab. J Am Coll Cardiol 2014; 63:2531-2540.

13- Stein E, Giugliano R, Koren M, Raal F, Roth $\mathrm{E}$, Weiss $\mathrm{R}$ et al. Efficacy and safety of evolocumab (AMG 145), a fully human monoclonal antibody to PCSK9, in hyperlipidaemic patients on various background lipid therapies: pooled analysis of 1359 patients in four phase 2 trials. Eur Heart J 2014; 35: 2249-2259

14- Sullivan D, Olsson A, Scott R, Kim J, Xue A, Gebski $\mathrm{V}$ et al. Effect of a Monoclonal Antibody to PCSK9 on Low-Density Lipoprotein Cholesterol Levels in Statin-Intolerant Patients The GAUSS Randomized Trial FREE. J Am Med Assoc 2012; 308:2497-2506.

15- Raal F, Scott R, Somaratne R, Bridges I, Li G, Wasserman $\mathrm{S}$ et al. Low-Density Lipoprotein Cholesterol-Lowering Effects of AMG 145, a Monoclonal Antibody to Proprotein Convertase Subtilisin/Kexin Type 9 Serine Protease in Patients With Heterozygous Familial Hypercholesterolemia The Reduction of LDL-C With PCSK9 Inhibition in Heterozygous Familial Hypercholesterolemia Disorder (RUTHERFORD) Randomized Trial. Circulation 2012; 126:2408-2417.

16- Raal F, Stein E, Dufour R, Turner T, Civeira $\mathrm{F}$, Burgess L et al. PCSK9 inhibition with evolocumab (AMG 145) in heterozygous familial hypercholesterolaemia (RUTHERFORD-2): a randomised, double-blind, placebo-controlled trial. Lancet 2015; 385: 331-340. 
17- Raal F, Honarpour N, Blom D, Hovingh GK, $\mathrm{Xu} \mathrm{F}$, Scott $\mathrm{R}$ et al. Inhibition of PCSK9 with evolocumab in homozygous familial hypercholesterolaemia (TESLA Part B): a randomised, doubleblind, placebo-controlled trial. Lancet 2015; 385 : 341-350.

18- Sabatine M, Giugliano R, Wiviott S, Raal F, Blom D, Robinson J et al. Efficacy and Safety of Evolocumab in Reducing Lipids and Cardiovascular Events. N Engl J Med 2015; 372:15001509.

19- Raal F, Giugliano R, Sabatine M, Koren M, Langslet G, Bays $\mathrm{H}$ et al. Reduction in Lipoprotein(a) With PCSK9 Monoclonal Antibody Evolocumab (AMG 145) A Pooled Analysis of More Than 1,300 Patients in 4 Phase II Trials. J Am Coll Cardiol 2014; 63:1278-1288.

\section{CONFLICTO DE INTERÉS Y/O AGRADE-} CIMIENTOS

Los autores declaran que no existió ningún conflicto de interés en el presente reporte. 